\title{
Virtual Electron Backscatter Diffraction for Multiscale Defect Characterization
}

\author{
Chaoyi Zhu ${ }^{1}$, Dylan Madisetti ${ }^{2}$, Jaafar El-Awady ${ }^{2}$ and Marc De Graef ${ }^{3}$
}

${ }^{1}$ Carnegie Mellon University, United States, ${ }^{2}$ Johns Hopkins University, United States, ${ }^{3}$ Carnegie Mellon University, Pittsburgh, Pennsylvania, United States

The plasticity of structural materials such as metals and alloys is dictated by motion and accumulation of curvilinear lattice defects, i.e., dislocations. Development of multiscale simulation of dislocations has enabled accurate modeling of dislocation interaction and multiplication across a wide range of time scales and length scales. Today, the research frontier of dislocation simulation is to use lower scale models at a fundamental level to inform higher scale "coarse grained" models. While dislocation simulations have celebrated their successes at their individual length scales, it is imperative to benchmark and calibrate these models so that we can connect them together to form a concrete multiscale framework [1]. In addition, a tool that accurately translates modeling results to experimentally observed features is also needed to further strengthen the multiscale hierarchy.

Historically, (scanning) transmission electron microscopes (S/TEM) were used to characterize local perturbations of lattice structure due to the presence of dislocations at the atomic scale [2]. This has also motivated the development of dynamical electron diffraction models to realistically account for the diffraction contrast observed experimentally [3]. Recently, scanning electron microscope-based techniques, such as the electron backscatter diffraction (EBSD) technique, have been used to characterize dislocation structures, allowing for statistically relevant quantification of dislocation evolution and patterning. More specifically, the lattice curvature measurement made possible by EBSD provides spatially resolved mapping of geometrically necessary dislocation (GND) density based on the continuum dislocation theory [4]. Despite a significant number of publications involving this method, the representation of discrete dislocations with a continuous dislocation density field in the context of material characterization is still loosely understood.

In this study, we present an idea for a virtual EBSD experiment to obtain critical information regarding dislocation characterization using EBSD, as shown in Figure 1. Within this approach, dynamical simulation of EBSD patterns is discretized into multiple depth bins, enabling an approximate deformation model to be imposed on depth specific EBSD patterns weighted by the corresponding Monte Carlo electron yield [5]. By convolving a finite interaction volume with three-dimensional simulation data, we can simulate diffraction patterns containing realistic deformation information at a specific length scale. In Figure 2, three types of example input data sets are used for the virtual experimentation carried out at 10 $\mathrm{kV}$ on a nickel sample. For the analytically derived single Yoffe dislocation data [6], we observe shape distortion of the deformation field along the longer axis of interaction volume. Additionally, the superposition of multiple dislocation deformation fields in the DDD simulation [7] produces a smoothly varying gradient in the representative volume. Finally, the virtual experiment shows intragranular kernel average misorientation (KAM) information from the CPFEM simulation [8] that is often correlated with non-uniform plastic deformation.

In summary, the virtual EBSD experiment provides a unique perspective to investigate dislocation characterization in EBSD. It allows researchers to understand the constraints in the characterization method and interpret experimentally collected data. More importantly. it can be used as a tool to further our understanding of dislocations across different length scales.

Funding Source: DoD Vannevar-Bush Faculty Fellowship (N00014-16-1-2821). 
Virtual EBSD Characterization

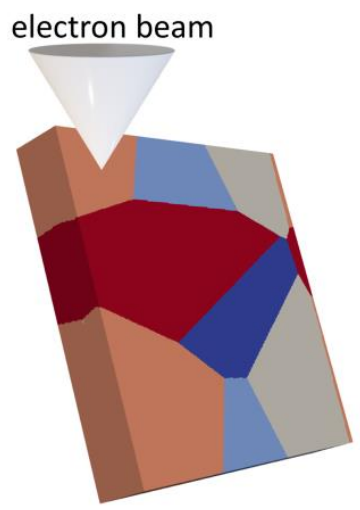

Interaction Volume Approach for EBSP Simulation

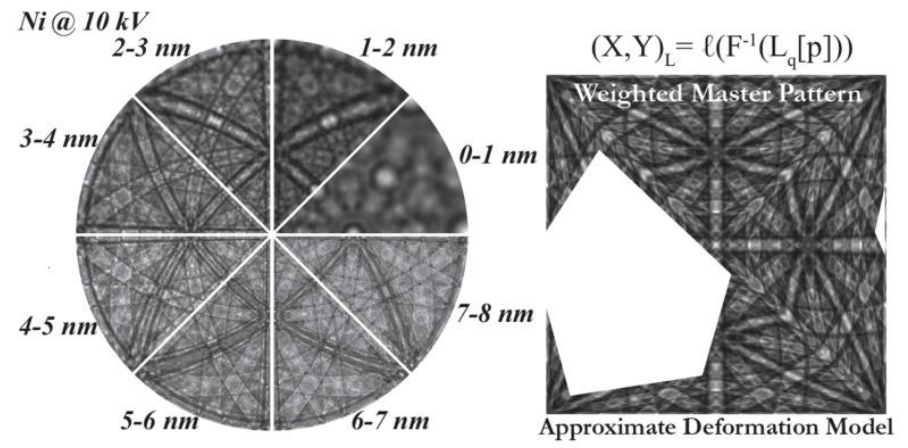

Electron Beam-Sample Interaction Volume

\begin{tabular}{|c|c|c|c|c|c|c|}
\hline Depth $=0-1 \mathrm{~nm}$ & Depth $=1-2 \mathrm{~nm}$ & Depth $=2-3 \mathrm{~nm}$ & Depth $=3-4 \mathrm{~nm}$ & Depth $=4-5 \mathrm{~nm}$ & Depth $=5-6 \mathrm{~nm}$ & Depth $=6-7 \mathrm{~nm}$ \\
\hline$A$ & 1 & 1 & $A$ & $A$ & & \\
\hline
\end{tabular}

Figure 1. Figure 1: Virtual EBSD characterization on a simulation data using the interaction volume approach for EBSP simulation.
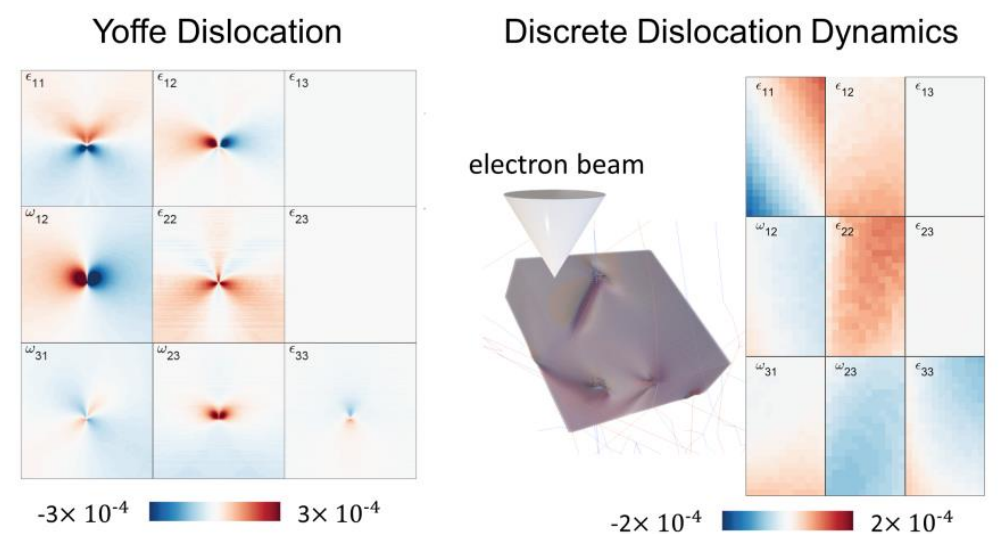

\section{Crystal Plasticity Finite Element Method}

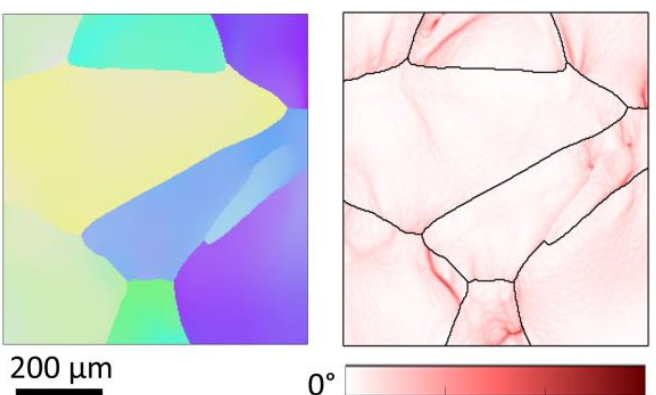

$1.5^{\circ}$

Figure 2. Multiscale EBSD virtual characterization on (left) an analytically derived Yoffe edge dislocation, (middle) a small volume extracted from a discrete dislocation dynamics simulation, (right) the surface of a crystal plasticity finite element, all based on nickel as test material at $10 \mathrm{kV}$.

\section{References}

[1] Bertin, N., Sills, R.B. and Cai, W., Annual Review of Materials Research, 2020.

[2] Hirsch, P.B., Horne,R.W. and Whelan, M.J., Philosophical Magazine, 1956.

[3] Howie, A. and Whelan, M.J., Proceedings of the Royal Society of London, 1961

[4] Nye, J.F., Acta Metallurgica, 1953

[5] Zhu, C. and De Graef, M., Ultramicroscopy, 2020.

[6] E.H.Yoffe, A dislocation at a free surface, Philosophical Magazine, 1961.

[7] Lavenstein, S., Gu, Y., Madisetti, D. and El-Awady, J.A., Science, 2020.

[8] Roters, F., Diehl, et al., Computational Materials Science, 20. 